MS07-P05

\section{Insights into the chromosome partitioning system, ParABS}

\author{
Yuh-ju sun ${ }^{1}$ \\ 1. Institute of Bioinformatics and Structural Biology, National Tsing \\ Hua University, Taiwan, Hsinchu, Taiwan \\ email: yjsun@life.nthu.edu.tw
}

Chromosome segregation is regulated by the ParABS system that is an important biological process in all domains of life. ParABS system includes ParA (an ATPase), ParB (a parS binding protein) and parS (a centromere-like dsDNA). The homologous proteins of ParA and ParB in Helicobacter pylori are $\mathrm{HpSoj}$ and $\mathrm{HpSpo0J}$, respectively. We characterized the binding of parS and $H p S p o 0 \mathrm{~J}$ and solved the crystal structure of the HpSpo0J-parS complex, with two HpSpo0J molecules bind with one parS. HpSpo0J interacts vertically and horizontally with its neighbors through the N-terminal domain to form an oligomer. These adjacent and transverse interactions might be needed for molecular assembly of a high order nucleoprotein complex and for ParB spreading. In addition, the ATPase activity of $\mathrm{HpSoj}$ was determined and the non-specific DNA binding of $H p S o j$ dimer was detected. Based on these findings, we propose a structural model for the HpSoj and HpSpo0J complex in the ParABS partitioning system.

References:

Proc. Natl. Acad. Sci. USA (2015), 112 (21), 6613-6618

Keywords: chromosome partitioning system, ParABS, segregation

\section{MS07-P06}

\section{Nanocrystallization of the Nucleoid of Bacteria under Stress}

Yurii Krupyanskii ${ }^{1}$, Natalia Loyko ${ }^{1}$, Ksenia Tereshkina ${ }^{1}$, Vladislav Kovalenko ${ }^{1}$, Eduard Tereshkin ${ }^{1}$, Olga Sokolova ${ }^{2}$, Alexander Popov ${ }^{3}$

1. Structure of Matter + Semenov Institute of Chemical Physics RAS, Moscow, Russia

2. Biological Faculty + Moscow State University, Moscow, Russia

3. ID-23-1 + European Synchrotyron Radiation Facility, Grenoble, France

email: yuriifkru@gmail.com

Structural, biochemical, and genetic changes caused by stress factors are known to be largely similar for cells of all modern organisms, which inherited the basic strategies of adaptation to different types of stress from their ancient ancestors. One of the strategies implemented in bacterial cells for constantly changing environmental conditions is related to the protection of the nucleoid from unfavourable environmental conditions by binding of DNA to specific histone-like proteins-DPS (DNA-binding protein from starved cells), in nanocrystalline complex, which helps to protect the nucleoid from damage and resume the activity of the bacterial cells later. The structure of these crystals within a cell was studied by use of synchrotron radiation on macromolecular crystallography station ID-23-1 as well as by use of transmission electron microscopy and tomography. However, the DNA conformation within these complexes has remained unsolved [1]. It was decided to decipher the structure of DNA-DPS crystals obtained in vitro which is in some respect the model of the structure of a nanocrystalline nucleoid. To this end crystalline complexes of DNA-DPS were studied with two different DNAs (one was linear and short DNA -24 b / p, the second was pBluescript SK (2958 $\mathrm{b} / \mathrm{p}$ ).) The crystal sizes reached $0.05 \mathrm{~mm}$. The structure of the crystals of the DNA-Dps complexes (the resolution of this structure reaches $2.6 \AA$ ) is different from that previously published in the RCSB Protein Data Bank database. The crystal structure of the DNA-DPS complexes was slightly different for the different DNA length. At first superficial glance, one can speak of the absence of DNA molecules in a given crystal. Positions of DNA in our crystals, probably, were chaotic; this point explains why we miss the DNA on the obtained electron density map. Obtained results will allow us to get closer in understanding of the protection mechanism of biocrystallization inside a living cell.

This work was supported within frameworks of the state task for ICP RAS 0082-2014-0001 (state registration \#AAAA-A17-117040610310-6).

References:

1. Sinitsyn D.O. et al., (2017). Biocrystallization of bacterial nucleoid under stress - Russian Journal of Physical Chemistry B, v. 11 , p. 833-838.

Keywords: biocrystallization, nucleoid, bacteria 